\title{
REVISIONES
}

\section{Un acercamiento a los movimientos de renovación pedagógica, a partir de las rupturas epistemológicas de los nuevos movimientos sociales}

\author{
An approach to the movements of pedagogic renewal seen from \\ the epistemological breakup of the new social movements
Uma aproximação aos movimentos de renovação pedagógica, a partir do rompimento epistemológico dos novos movimentos sociais

\begin{abstract}
Pedro Moscoso
Alumno doctorando en Procesos de Cambio Social. Facultad de Educación y Trabajo Social, Universidad de Valladolid, Paseo de Belén, 1.47006, Valladolid, España. E-mail: pemf28@gmail.com
\end{abstract}

\section{RESUMEN}

El sentido del presente ensayo es el de realizar un ejercicio reflexivo respecto a las implicancias epistemológicas que posee la aparición de los Nuevos Movimientos Sociales (NMS) en los Movimientos de Renovación Pedagógica (MRP). Será de especial interés establecer cómo estas nuevas miradas teórico-conceptuales respecto a los movimientos sociales permiten pensar en un discurso educativo, ligado íntimamente con procesos de formación identitaria y cultural. La apuesta es lograr articular los principios conceptuales de los NMS con los MRP, para establecer su posibilidad real de responder a una nueva alternativa educativo-social.

Palabras clave: sujeto social, identidad, cultura, poder, educación.

\begin{abstract}
The aim of this essay is to make a reflective exercise about the epistemological implications that the appearance of the New Social Movements (NSM) has had on the Movements of Pedagogic Renewal (MPR). It will be of particular interest to establish how these new perspectives on theoretical and conceptual social movements allow us to think of an educational discourse, intimately linked to processes of identity y cultural formation. The goal is to articulate the conceptual principles of the NMS with the MPR, so to establish the real feasibility to cope with a new socio-educational alternative.
\end{abstract}

Key words: social subject, identity, culture, power, education.

\section{RESUMO}

O sentido do presente ensaio é fazer um exercício reflexivo sobre as implicações epistemológicas que o surgimento dos Novos Movimentos Sociais (NMS) tem nos Movimentos de Renovação Pedagógica (MRP). Será de especial interesse estabelecer a forma como essas novas perspectivas teórico-conceituais dos movimentos sociais permitem-nos pensar num discurso educacional intimamente ligado aos processos de formação cultural e da identidade. O objetivo é articular os princípios conceituais dos NMS com os MRP para estabelecer a sua real possibilidade de responder a uma nova alternativa educativo-social.

Palavras-chave: sujeito social, identidade, cultura, poder, educação. 


\section{PROLEGOMENOS}

“Una realidad normal se hace difícil de conocer y analizar...”. Godelier

El presente escrito intentará analizar la problemática planteada por los Nuevos Movimientos Sociales (NMS). Con esto, nos referimos a una nueva forma de entender la realidad sobre lo social, a partir de la inclusión de nociones tales como la construcción de significados compartidos y la necesidad de generar vinculaciones, ya no articuladas directamente con los problemas planteados por el materialismo histórico, basadas en grandes cambios provocados por la modernidad. Lo anterior posibilita la emergencia de una serie de movimientos, con un carácter de convocatoria cultural, que buscan respuestas a problemas que el devenir moderno ha planteado, y ligados a su imposibilidad de cumplir con la promesa tecnocientífica. Se abren así nuevas configuraciones de sentido, plurales y diversas, alejadas de las categorías cartesianas clásicas, que ponen en conflicto a las teorías sociológicas tradicionales, en la medida que no logran abarcar desde sus desarrollos teóricos los fenómenos sociales.

Para lograr lo anterior, creemos necesario retomar los elementos conceptuales que impiden configurar el fenómeno al concepto. Esto es de suma importancia, ya que creemos que el intento de configurar una mirada "alternativa" respecto a los movimientos sociales responde a un nuevo espacio de acontecimiento de lo social, entendiendo esto como posibilidad de aparición de una alteridad -frente a la marginación de los límites impuestos de un modelo político tradicional-. Nuestra propuesta de análisis se sostiene en que mientras más se radicaliza el discurso de lo social, lo que se logra no es un quiebre en la dinámica de la política, sino, por el contrario, una negación que la afirma. Esto quiere decir que las resistencias actuales estarían integradas dentro de los discursos de la política, por lo que no vienen cargados con la sorpresa, el desvío, la discontinuidad, sino que, al contrario, son parte de la reproducción de la hegemonía.

Esto no quiere decir que no sea importante considerar factores económicos o políticos, que posibilitan la emergencia de determinado movimiento, sino que pensamos que el análisis de dichos movimientos sirve como espacio de posibilidad para abordar dichos fenómenos desde su particularidad, alejándose de la pretensión universalizadora de los modelos clásicos. Conceptos tales como el de "actor social" o "subjetividad colectiva" permiten a los Nuevos Movimientos Sociales establecerse desde la búsqueda de individualidad y la identidad concomitante, poniendo en juego elementos ligados a espacios de construcción potencial que, presumiblemente, permitirán la aproximación a la realidad desde el alejamiento del sentido de las instituciones tradicionales. Es decir, pensar el proyecto de los NMS como espacio de aparición de lo otro. El resultado de esto, podríamos pensar, dice relación con que la aparición de esta nueva configuración viene a cuestionar, en sí misma, los mecanismos y las estructuras institucionales que sostienen el discurso económico-político-científico basado en una racionalidad neoliberal. Asistimos, de esta manera -podríamos decir que estamos viviendo- a un punto de inflexión en que los saberes modernos caen, entran en crisis y han perdido sus referentes.

Para lograr dicho cometido, pretendemos hacer un recorrido descriptivo respecto a la noción de NMS, definiendo sus principales características, líneas de acción y diferencias del modelo clásico. Luego, a partir de una mirada epistemológica, nos referiremos al 
problema de la identidad, la dimensión cultural y la configuración de nuevos sentidos. A partir de esta cartografía, pretendemos centrar la mirada sobre los Movimientos de Renovación Pedagógica (MRP), como espacios que promueven nuevos modos de abordar la educación, fundados en corrientes pedagógicas críticas, que hacen especial hincapié en la necesidad de re-entender la educación como espacio de reflexión, formación ciudadana y democracia, alejándola del modelo funcionalista tecnocrático que impera actualmente.

Las implicancias que tiene esta nueva configuración sobre la educación, en tanto espacio social central para la canalización de información, manejo de códigos culturales y espacio institucional de socialización, permite suponer que existen relaciones simbólicas que es necesario investigar, tomando como elemento central la constitución de la identidad y los aspectos culturales asociados a éstos. Se trata de develar si es que efectivamente se producen espacios de interacción dialógica entre ambos y se dejarán planteadas ciertas inquietudes que pudiesen servir para establecer una reflexión profunda de los cambios por venir.

\section{LA CUESTION DE LOS NUEVOS MOVIMIENTOS SOCIALES (NMS)}

Los nuevos movimientos sociales surgen a finales de los años 60 en Europa Occidental y Estados Unidos. Éstos han sido ideológicamente agrupados según la tradición política de izquierdas y caracterizados por el rechazo a los regímenes autoritarios. Estos movimientos hacen tomar conciencia sobre las amenazas que ha creado el hombre, a partir de aquellos elementos considerados característicos de la modernidad, tales como los efectos de la tecnoeconomía sobre la naturaleza, los graves deterioros ecológicos, las políticas de exclusión y marginación, etc. (Laraña, 1999). Son movimientos que reaccionan frente al sistema social, cultural, económico y político, al mostrar sus contradicciones y su incapacidad para resolver los problemas que el propio modelo establecido origina.

Estos movimientos son portadores de una gran sensibilidad respecto de las relaciones sociales y políticas. Muestran dónde están las contradicciones o conflictos sociales fundamentales de una sociedad con pretensión de modelo universal y se convierten en agentes sociales en pro de la superación de las mismas. Ponen en entredicho el proceso de racionalización de Occidente, señalado por Weber, ligado a un modo funcionalista de ver el mundo, a las motivaciones éticas y a un comportamiento disciplinado y competitivo. Desde esta perspectiva, se señala la dificultad de sostener, dentro de este contexto, la idea de progreso como proceso histórico continuo, planteado por la modernidad (Mardones, 1996).

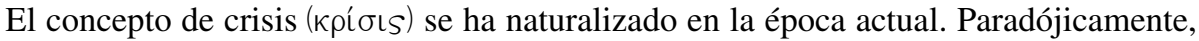
ha pasado a transformarse en un concepto ligado al estado natural de las cosas, cuando etimológicamente la noción de crisis hace referencia a un momento en que se produce un cambio muy marcado en algo. Frente a esto, creemos que el funcionamiento discursivo moderno remite a una serie de cambios parciales, a través de las reglas discursivas que operan como dispositivos de poder, que permiten que el paradigma perdure y se estabilice en el cambio. Así, hace sentido la idea según la cual "Si queremos que todo siga como está, es preciso que todo cambie...” (Tomasi de Lampedusa, 2003).

Los NMS invocan el malestar de la cultura, entendido como el precio a pagar en beneficio de la modernización. Lo anterior lo podemos caracterizar a partir de una pérdida de 
referentes, que antiguamente aseguraban la coherencia y el orden de las sociedades. Por lo tanto, el problema que buscan abordar los NMS se encuentra relacionado a uno de visión de mundo, de lógica, de valores, comportamientos y estilos de vida. Estos suponen un cambio cualitativo, que tienda hacia una mejora del ser y no del tener (Zubero, 1996).

El contexto de aparición de los NMS remitiría, en definitiva, a sociedades pobres de sentido, en las que el hombre se ha comenzado a pensar como una célula reaccional del proceso mercantil. Dentro de esta lógica cobra sentido el problema de la identidad individual, que acontecería vinculada y cosificada por el proceso de racionalización, imponiendo determinados parámetros de normalidad-anormalidad. Es decir, la pérdida de sentido se encuentra íntimamente ligada a la incorporación de una identidad "individualizadora", que permitiría situar el problema de búsqueda de sentido en referencias externas, es decir, el momento en que la vida se transforma en una búsqueda incesante de referentes con los cuales identificarse.

A diferencia de los enfoques tradicionales, los NMS hacen resaltar la importancia de los factores simbólicos y culturales. Las nuevas formas de acción colectiva están ligadas a cuestiones de identidad individual y colectiva, ideas, valores y significados de los actores sociales para entender su participación, con metas centradas en el desarrollo personal y cambios en las formas de interacción. Contrastan, en este sentido, con las visiones tradicionales -estructuralistas- de movimientos sociales, centrados en el conocimiento de la base económica, de la clase del movimiento o del estatus social de los seguidores que situaban al grupo en la estructura social, es decir, como una respuesta a un sentido de injusticia especificado por la ideología, que suministraba el impulso para la movilización (Zubero, 1996).

Dentro de las características principales de los NMS, nombramos las más relevantes. En primer lugar, éstos no tienen una relación clara con los roles estructurales de sus seguidores, trascendiendo la estructura de clase y poseyendo un estatus social difuso. Se caracterizan por el pluralismo de ideas y valores; suelen ser pragmáticos y persiguen reformas institucionales que amplíen los sistemas de participación en decisiones de interés colectivo. En su significado político, implican una democratización de la vida cotidiana y expansión de las dimensiones civiles. Además, implican el desarrollo de nuevos aspectos de la identidad de sus miembros, orientados hacia un sentido de pertenencia, que determinará la imagen que sus miembros tienen de sí mismos y los nuevos significados a su vida cotidiana que se construyen a partir de forma colectiva. Lo anterior produce una difuminación de la relación entre el individuo y el grupo. Por último toman formas flexibles, cambiantes y poco profesionalizadas, implicándose en aspectos íntimos de la vida humana (Laraña, 1993).

\section{ASPECTOS EPISTEMOLOGICOS EN TORNO A LOS NUEVOS MOVIMIENTOS SOCIALES}

Como ya comentamos en el apartado introductorio, los NMS plantean la necesidad de realizar un replanteamiento epistemológico para la investigación, dándole un nuevo énfasis a conceptos tales como el de sujetos colectivos, actores sociales y subjetividad colectiva. Esto implica necesariamente preguntarse por las formas en que se construye el conocimiento, en especial respecto al posicionamiento político de las ciencias sociales en la producción del conocimiento (Retamozo, 2006). Implica, además, asumir una noción 
de sujeto alejada de la imperante en los modelos clásicos, dejando fuera las dimensiones culturales y cognitivas. Se podría hablar de una suerte de descolonización de los mecanismos discursivos modernos.

Para entender esta nueva mirada, consideramos la noción de sujetos como condensadores de historicidad con posibilidad de futuro (Zemelman, 1992). Esto requiere entender la subjetividad, no como una estructura estática objetivable, sino como productosproductores de la sociedad. Además, implica asumir la posibilidad de un sujeto que se hace parte de los procesos de construcción del conocimiento, que es capaz de revelarse y construirse en estos procesos y del lugar que este ocupa en la producción de la (su) historia. Por último, implica entender el mundo social como una construcción social. Esta visión epistemológica, desde la complejidad crítica, plantea un vuelco a la idea de realidad, ahora entendida desde el movimiento constante, incorporando la conciencia histórica como dimensión fundamental del pensamiento y articulando las relaciones entre cultura y el conocimiento con los procesos particulares de dominación y subordinación social (Martínez, 1990).

Por su parte, la noción de subjetividad colectiva contiene la cuestión respecto a la forma en que se construyen y conforman los sentidos. Esto remite a la noción de hegemonía como espacio de conflicto (Broccoli, 1977). La cultura, como un campo de lucha social, en cuanto el dominio no se ejerce sólo por la fuerza, sino también por el consentimiento, a menudo disfrazado de "sentido común". El pensamiento hegemónico es aquello que parece natural, sobre lo que ni siquiera se piensa, que es construcción histórica y, por tanto, cambiable.

El proyecto moderno ha desarrollado un sistema de control social a partir de la configuración del sentido de la acción individual, ligado a aspectos que tradicionalmente eran considerados como privados (cuerpo, sexualidad, relación afectiva), subjetivos (problemas cognitivos y emocionales, motivos, deseos) o inclusive biológicos. El aparato tecnocientífico ha detentado el poder sobre estos campos. Así, acontecen cambios en los modos de control sobre la población, en que la violencia se vuelve difusa y se hace omnipresente.

El filósofo francés Michel Foucault plantea, al respecto, que la vida del individuo está sometida a una Biopolítica, donde un conjunto de prescripciones y naturalizaciones se apoderan de la superficie de los cuerpos, construyendo y disponiendo un sentimiento normativo sobre ellos (Foucault, 2007). Por lo tanto, pensamos que estos nuevos regímenes de control han configurado un corpus de representaciones de un cierto "orden", centrado en lo político. Un orden que ha desplazado cualquier posibilidad de articulación subjetiva de proyecto político desde lo social. El poder, desde esta perspectiva, se entiende como intrínseco a la naturaleza misma de las cosas, naturalizando los hechos sociales y generando un efecto de inevitabilidad frente a determinados procesos y cambios.

Asistimos a una concepción determinista de la realidad social, a partir de un conocimiento social objetivado y objetivante, de carácter explicativo, eliminando los elementos culturales particulares. El ideal de este principio de legitimación reside en la posibilidad de ocultar su carácter de realidad construida. En caso de que los mecanismos de legitimación fracasen, entran en juego los mecanismos de control social. Lo anterior, asociado a la idea de quién controla el poner nombre es quien controla la realidad (Zubero, 1996).

En este sentido, los NMS se constituirán alrededor de un mensaje que impugna el hecho de que los aparatos tecnoburocráticos intenten imponerse a los acontecimientos 
individuales y colectivos. Hay un tema ligado a la reapropiación de la identidad, es decir, la posibilidad de construir el significado de lo que los sujetos son y lo que hacen (Melucci, 1994).

Específicamente, se constituyen como un cuestionamiento de sentido. La acción del movimiento social tiene una función de denuncia, o dicho de otra manera, de develar lo que enmascara determinados intereses y formas de poder. Cuestionamiento que pide cuentas, en definitiva, a los modelos culturales hegemónicos que han impuesto categorías conceptuales, morales y valores que rigen los comportamientos y actitudes cotidianas de las personas.

Por lo tanto, podemos localizar sus bases epistemológicas en el nivel de los modelos culturales, allí donde se juegan la identidad personal y el sentido de la vida. Como señalaría Habermas, el problema es de estilo o gramática de la vida, entendiendo que los conflictos ya no se producen en el ámbito de la reproducción material, sino que surgen en el ámbito de la reproducción social, la integración social y socialización. Es decir, operaría una cosificación de los ámbitos de acción comunicativos, a los que ya no se puede hacer frente a través de los medios, dinero y poder (Habermas, 1987). Por lo tanto, el problema moderno girará en torno a la identidad y el sentido de la vida.

En síntesis, el problema se centrará en la defensa de la identidad, es decir, a partir de la posibilidad de poder llamarse a sí mismos como les resulte conveniente, logrando mantener así una imagen integrada y duradera. Al respecto, Touraine señala que "Lo que orienta tanto la acción colectiva como las pasiones individuales no es la construcción de un mundo prometeico, de una sociedad justa, menos todavía la conquista de libertades públicas para el derrocamiento de un Antiguo Régimen, sino la afirmación del derecho de cada individuo a crear y regir su propia individualidad" (Touraine, 1987: 12). En este sentido, plantea la identidad como un dispositivo orientado a rechazar la definición social de los roles institucionalizados que debe ocupar el actor social.

Así planteado, los NMS exigen la necesidad de replantear ciertos elementos que parecían claros en el análisis sociológico tradicional. Melucci se refiere al problema epistemológico, entendiendo la acción colectiva como un resultado y no como punto de partida. Esto coincide con una desontologización de la noción de movimiento social, es decir, la comprensión de los movimientos sociales como procesos en que los actores sociales producen significados, se comunican, toman decisiones e invierten emociones, y no como una realidad estática desde la cual se puedan predefinir ni delimitar sus objetivos. Esto requiere de un doble ejercicio analítico: por un lado, la deconstrucción (disección) en cuanto a los orígenes sociales del movimiento y sus creencias; por otro, una reconstrucción (Melucci, 1994).

En este sentido, el autor se enfoca en la noción de acción colectiva, "considerada como el resultado de intenciones, recursos y límites, con una orientación construida por medio de relaciones sociales dentro de un sistema de oportunidades y restricciones" (Melucci, 1994: 42). Es necesario entenderla como el resultado de múltiples procesos que favorecen o impiden su formación y el mantenimiento de sus estructuras, es decir, como producto de procesos sociales diferenciados, de orientaciones de acción, de elementos de estructura y motivaciones que pueden ser combinadas de distintas maneras. En resumen, se entienden los fenómenos colectivos como procesos en los cuales los actores producen significados, comunican, negocian y toman decisiones.

A lo anterior, el autor agrega la importancia de considerar la "identidad colectiva", como posibilidad para la realización de las expectativas de los actores sociales que 
componen el movimiento. Atañe, además, a las orientaciones de acción y al ámbito de oportunidades y restricciones en el que tiene lugar la acción, en un proceso que se construye y negocia mediante las relaciones que unen a los individuos:

"La identidad colectiva es, por lo tanto, un proceso mediante el cual los actores producen las estructuras cognoscitivas comunes que les permiten valorar el ambiente y calcular los costos y beneficios de acción; las definiciones que formulan son, por un lado, el resultado de las interacciones negociadas y de las relaciones de influencia y, por el otro, el fruto del reconocimiento emocional" (Melucci, 1994: 66).

Por lo tanto, el interés de un individuo para implicarse en la relación colectiva estará mediado por la capacidad diferencial para definir una identidad, es decir, participar en los procesos de construcción de identidad.

Así se explica cómo los NMS son fenómenos que surgen para responder a conflictos propios de las sociedades capitalistas postindustriales. Dichos conflictos escapan al ámbito del tradicional sistema económico-industrial y se desplazan hacia espacios culturales, a partir de mecanismos de control sobre los mismos recursos fundamentales que permiten su funcionamiento. Esto remite, en definitiva, a un poder que afecta la vida cotidiana, las motivaciones de la acción individual y el proceso por el cual las personas dan significado a las cosas y a sus acciones (Foucault, 1993).

Como ya comentamos anteriormente, el sociólogo francés Alain Touraine señala que los movimientos sociales deben ser comprendidos desde la perspectiva del "actor social", entendiendo a este último como promotor de situaciones históricas. Desde esta lógica, el actor deberá ser capaz de desprenderse de las formas y normas de reproducción de los comportamientos, para poder así participar en la producción de nuevos modelos culturales (Touraine, 1987). En este sentido, la posición del autor consiste en abandonar las perspectivas de lo social como funcionamiento, entendiendo que éste se constituye a partir de la suma de los deseos e intereses individuales:

"El regreso del actor no se realiza de modo conquistador sino defensivo; no convoca más a cada individuo para fusionarse en un vasto impulso colectivo, sino por el contrario en uno de signo anticolectivista. Se niega a endiosar a la sociedad y más aún al Estado. Cree más en las libertades personales que en la liberación colectiva, al afirmar que la vida social no se rige por leyes naturales o históricas sino por la acción de los que luchan y negocian para brindar cierta forma social a las orientaciones culturales que aprecia" (Touraine, 1987: 39).

\section{EMERGENCIA Y BASES DE LOS MOVIMIENTOS DE RENOVACION PEDAGOGICA}

Los Movimientos de Renovación Pedagógica surgen en el contexto de un mundo globalizado, caracterizado por un cuestionamiento de los paradigmas de la educación clásica, centrados en una didáctica funcional que deja fuera aspectos ligados al contexto, la historia y la dimensión política de la misma. En este sentido, señalamos que la crisis de los sistemas educativos, bajo criterios de eficacia y competitividad, ha llevado a la implantación de las nuevas reformas educativas, de carácter fuertemente neoliberal, en las que la palabra calidad se asocia a selección y competitividad en su vertiente profesionalista, y a la domesticación, desinformación y alienación en el campo de los valores (Llorente, 2005). 
La globalización ha generado nuevos efectos en todo ámbito, con un creciente proceso de racionalización basado en una mirada administrativa, con una fuerte función económica y socializadora. Ha generado hegemonías impuestas, lenguajes preeminentes (especialmente visuales), mecanismos operativos mentales que promocionan una determinada manera de procesar la información y pensar, y unos códigos comunicativos, unos símbolos y estereotipos que unifican y borran esa especificidad necesaria desde la que "mirar al otro", ya que no hay posibilidad de contraste (Antón, 2003).

Por lo tanto, nos podemos referir a una globalización cultural que implica a las distintas esferas sociales, entre ellas la educación. Todo esto, dentro de la denominada sociedad de la información, en que los medios de comunicación y nuevas tecnologías juegan un papel fundamental en la redefinición de identidades individuales y colectivas. Se produce una "planetarización del sistema", en que la unión planteará nuevos problemas respecto al control, circulación e intercambio de información, produciéndose una mundialización de los problemas y conflictos. En este contexto la localización territorial devendrá secundaria respecto al impacto simbólico sobre el planeta (Melucci, 1994).

Lo anterior permite agrupar una serie de acontecimientos aislados dentro de un marco, y reinterpretarlos dentro de un campo de acción delimitado por las corrientes progresistas y funcionalistas. Esto tendrá un efecto de reificación de la información, a partir del cual los dispositivos de poder se centrarán, ya no en el control sobre los contenidos, sino en los marcos de producción e interpretación de dicha información. En otras palabras, asistimos a un espacio de regulación de los campos de simbolización y decodificación de la información, que se verán desplazados desde las grandes organizaciones a la vida cotidiana, a partir de la intervención sobre medios para la construcción de una identidad estable, motivaciones, deseos e intereses. En este aspecto, pensamos, cobra relevancia el espacio educativo.

En el ámbito educativo, lo anterior se puede representar a partir de una banalización de temas referidos a la formación individual y colectiva, al aparecer en los currículos como temas transversales que nadie sabe muy bien dónde y cómo tratar, en medio de la fuerte especialización en áreas y la dictadura de los tiempos y espacios escolares. Valores que han sido cooptados por el sistema educativo, pervirtiendo su significado inicial y adaptándolos a las tesis neoliberales de la competición y el libre mercado (Llorente, 2005).

El tratamiento de los valores ha sido dejado de lado, o mejor dicho transformado, al insertarlo dentro de un régimen de competencias blandas, con la pretensión de poder objetivarlas y evaluarlas. Esto ha posibilitado la aparición de sistemas de evaluación especiales para dichos fines, universalizando valores con pretensión de verdad que excluyen sus contextos de aparición y funcionamiento. El efecto de lo anterior, es la producción de un modelo de valores que reniega la necesidad de cuestionamiento al modelo político-social. Se produce así una "apropiación de temas", por parte del poder dominante (económico y político), des-cargándolos de su contenido y potencialidad crítica.

A lo anterior, se le suma una excesiva confianza en las teorías educativas modernas, heredadas desde otras disciplinas científicas, lo que las convierte en espacios incompletos o doctrinarios. Schwab plantea esta situación como una "huida del objeto del propio campo", por lo que propone un nuevo enfoque epistemológico centrado en la deliberación metódica, en la generación de espacios comunicativos fluidos y negociados, a través de los cuales se puedan comprender los problemas concretos y reales de la práctica escolar, mediante un intercambio de significados e interpretaciones (Martínez, 1990). 
Frente a esto, surgen los Movimientos de Renovación Pedagógica, con el objetivo fundamental de recuperar la educación como derecho y servicio público que se debe garantizar desde los Estados y la escuela, entendida como lugar en el que se crea cultura. Esto reabre el debate ético necesario sobre educación, democracia y culturas. La escuela, en este sentido, es un escenario que permite un cuestionamiento de los modelos sociales hegemónicos y que puede dar paso a otros alternativos.

Los MRP tienen una fuerte base en las formulaciones planteadas por el discurso pedagógico crítico, como un intento de comprensión del mundo y de transformación de la realidad. Orientación crítica que cuestiona los presupuestos de orden social y educativo existentes, indagando en las relaciones existentes entre saber-identidad-poder. Lo anterior, por ejemplo, al trabajar sobre nociones tales como la de concientización, en el sentido conferido por Paulo Freire y tantos educadores de América Latina, que supone la educación como "un acto de conocimiento y un proceso de acción transformadora sobre la realidad" (Antón, 2003: 5). La perspectiva crítica cifra su fuerza en la convergencia de los movimientos educativos, pedagógicos, culturales, sociopolíticos e históricos.

Surge así la concepción de una "educación emancipatoria", que tiene como sentido fundamental la construcción de la propia identidad personal y social. Se dirige a que los educandos sean conscientes de su realidad, que aprendan a identificar sus emociones y sentimientos, a socializar desde el respeto a la diferencia, a adquirir competencias de pensamiento y acción que les permitan desenvolverse con capacidad crítica en el mundo (Llorente, 2003). Educación emancipatoria, al permitir crear escenarios de realidad alternativos que cuestionen la legitimidad del consenso y orden social vigente. En resumen, supone una corriente contracultural alternativa y contrahegemónica respecto al poder.

"Los MRPs se han enfrentado a la voluntad de poder en la escuela para favorecer la voluntad de vivir (...) han cuestionado la reproducción acrítica y propuesto la reflexión y comprensión crítica de la experiencia. Se pensó la alianza con el niño y la naturaleza y se buscó liberar al sujeto de vivir el placer de saber como una obligación. Y se cuestionó un espacio que aísla, unas relaciones que aburren o un tiempo que separa la vida en fragmentos inconexos" (Martínez, 1990).

Desde su creación, los MRP han trabajado incansablemente en la construcción de la escuela pública, popular y democrática, asumiendo un compromiso en la transformación social. Para ello, han profundizado en la participación social y en la organización democrática, a partir de la lucha contra la manipulación de los medios de comunicación de masas, la banalización de grandes problemas mundiales como la pobreza, la injusticia, la destrucción del medio ambiente o la represión. Además, han buscado superar la tendencia creciente a la desmovilización social, al consumismo salvaje y al ocio programado. Se inicia, así, una propuesta global de trasformación educativa, con carácter pedagógico y político, a través de la contrastación teoría-práctica, del desarrollo de un pensamiento práctico y mediante una metodología de trabajo participante y cooperativa (Llorente, 2003).

El carácter social y pedagógico de este movimiento educativo ha basado su quehacer fundamental en la existencia de comunidades críticas de profesores que, preocupados por mejorar el sistema educativo público, hablan de la Escuela Pública como un proyecto que es necesario definir y construir mediante la reflexión, debate e intercambio de 
experiencias, favoreciendo el debate profesional y social sobre lo que significa una enseñanza pública de calidad. Han tejido así una amplia red de intercambio y elaboración de alternativas pedagógicas, tanto en su dimensión educativa en relación a la tarea docente, como en su dimensión política, elaborando alternativas generales del modelo educativo e intentado incidir en los procesos generales de reforma, cambio político y educativo (Dómenech, 2003). Han tratado, con su práctica cotidiana, de superar la dicotomía entre teoría y práctica, a través de propuestas educativas bien fundamentadas, reclamando el control sobre su proceso de trabajo, devaluado como consecuencia de la fragmentación organizativa y curricular, del aislamiento, de la autonomía ficticia y de la rutinización y mecanización laboral.

El carácter político de estos movimientos refiere a la posibilidad de despertar a los individuos de su opresión y generar acciones de transformación social. A través de la educación se genera un espacio de construcción de sentidos que, hasta el momento, han sido regulados por los modelos de poder, a partir de la producción de un modelo de pensamiento único al servicio de las estructuras económicas que determina al sujeto, no solo en sus dimensiones ligadas a la adquisición de un corpus de conocimientos, sino también en sus dimensiones subjetivas. Los MRP proponen una relación entre educación e ideología, educación y producción: de ahí la importancia de reivindicar el papel del contenidos en las materias, que ha sido abandonado por las corrientes funcionalistas, entendiendo la educación como posibilidad de transmisión ideológica, de las relaciones sociales en la escuela, la reproducción social como reproducción cultural, mediante la imposición y ocultación, producción y consumo de recursos simbólicos (Melucci, 1994).

En este marco, la renovación pedagógica busca fines globales, tales como la creación de espacios de libertad o la liberación en lugares tan concretos como el aula. Por eso tiene sentido decir que se piensa globalmente y se actúa localmente. "Los MRPs, en mi opinión, representan (o deben representar) una plasmación, en la práctica, del principio tantas veces repetido de pensar global y actuar local. ¿Qué es sino poner en marcha experiencias, alternativas en el aula o el centro, conectadas con propuestas educativas y sociales globales y fines sociales generales?” (Aragón, 2003:27).

Desde esta perspectiva, se propone una articulación entre la educación crítica y una opción cultural. Por tanto, la cultura debe ser entendida como transformadora, es decir, como aquella que ayuda a comprender y actuar críticamente en la sociedad en la que vivimos, para superar la desigualdad y la dominación. Es la que conecta la reflexión con la acción, conectando la utopía con las realidades concretas que necesitan superarse y favoreciendo la participación crítica. En este sentido, la cultura transformadora conecta a los diferentes movimientos sociales entre sí, va construyendo -más que definiendo, aunque también- algunas claves comunes y va estimulando el enriquecimiento mutuo, mediante la diferencia de temas, pero también de formas de actuar, e incluso de ideas. Por último, implica entender la cultura, no como algo externo a los sujetos, sino como algo que se construye y se vive. En este sentido, se hace necesario considerar las relaciones entre saber-poder.

En síntesis, se hace un énfasis en la necesidad de integrar lo educativo y lo social, específicamente en su vertiente cultural. De acuerdo a esta visión, el campo educativo se puede ver enriquecido con visiones externas y con la creación de contextos críticos, así como con la asunción social de la educación como tema público. Los movimientos sociales, por su parte, se verían estimulados a tener más en cuenta su vertiente educativo- 
cultural dirigida a toda la población, así como a participar en la construcción progresiva pero constante de herramientas y contextos culturales transformadores (Ibáñez, 2003).

\section{REFLEXIONES PROVISIONALES}

A partir de lo anterior, se pueden generar las siguientes reflexiones, como parte de un análisis que requiere mayor exhaustividad, pero que preliminarmente nos deja algunas inquietudes.

Como primer elemento, se puede hablar de cómo los NMS nos abren nuevos espacios de reflexión en torno a cuestiones que revisten una importancia fundamental a nivel epistemológico. Esto, ligado a la cuestión de la identidad y la exploración del sí mismo, cuestiones que responden a emociones y ámbitos no reductibles a la racionalidad instrumental. Esto requiere modificar la mirada y ponerla sobre la posibilidad de redescubrir una alteridad incurable (el otro, lo otro, lo sagrado). Hay algo que escapa al poder de la racionalidad, que se encuentra más cerca de un espacio de silencio que se sustrae al flujo de la comunicación codificada, en la aparición de un acontecimiento propio de carácter local y específico, tomando como referente la idea de sujetos colectivos históricamente determinados. No hay, pues, una intención de generar categorías universalizantes ni extrapolables a todo ámbito de acción. Hay un movimiento de posibilidades de sentido, una búsqueda de espacios para, como dice Touraine, poder generarse identidad. Esto, lejos del poder que se ejerce sobre el control de los códigos (conjunto de reglas formales para organizar el conocimiento) que limitan el campo conceptual y los parámetros de realidad. Existiría una necesidad de reintegrar la experiencia humana, reconociendo la alteridad y el límite con arreglo a un principio de unidad. Un silencio y un retraimiento hacia un espacio interior, donde las palabras que quedan son aquellas que uno se dice a sí mismo. Tienen una extraordinaria fuerza de subversión simbólica.

El análisis que hemos planteado se sustenta en una nueva manera de comprender el poder, en una dimensión productiva, que se encuentra operando en todas partes, traspasando las barreras de la corporalidad para instalarse en nuestro deseo, en nuestros ideales y sentidos de vida. Esto hace del poder un elemento mucho más peligroso e incisivo. Sin embargo, de esta misma manera, los movimientos sociales parecen haber perdido su carácter de material para hacerse más inasibles, menos categorizables y más difusos. Como el sentido de los NMS responde a cuestiones ligadas a la existencia humana, que conciernen a todos, en la necesidad de hacer pública la construcción sobre lo privado, el Estado se encuentra interpelado por una llamada a la que no puede responder desde sus actuales modelos de realidad.

El núcleo antagonista del movimiento radica, por tanto, en su capacidad de resistencia y de subvertir los códigos dominantes. En este sentido, los MRP se encuentran seriamente implicados, ya que se mueven en espacios donde se producen y reproducen los códigos dominantes. Se anticipa así la posibilidad de nombrar de modos otros el espacio y el tiempo, a través de la construcción de nuevos lenguajes que cambian las palabras empleadas en el orden social. Lo anterior nos hace pensar en la idea de una sabiduría que integra el conocimiento ligado a la experiencia particular, local, históricamente determinada, que será una tarea decisiva para estos movimientos. La posibilidad de constitución del actor colectivo, como el modo de constitución de su propia subjetividad-solidaria. 
Entender los MRP desde la perspectiva integradora de una cultura transformadora, implica pensar en ellos como movimientos sociales de carácter macro, que apelan a la integración de otros movimientos sociales. En este sentido, es posible pensar en una función ética de los MRP, alejada de la prescripción moral impuesta por los códigos normativos modernos. Implica la formación ciudadana a partir de una nueva forma de entender lo que es ser sujeto social.

\section{REFERENCIAS BIBLIOGRAFICAS}

Antón, J.A. (2003). "La Pedagogía Crítica desde la Perspectiva de los Movimientos Sociales". Tabanque, Revista Pedagógica, $\mathrm{N}^{\circ}$ 17. Pp. 51-70. Universidad de Valladolid, Valladolid.

Aragón, M.A. (2003). "Los Movimientos de Renovación Pedagógica y la transformación socioeducativa". Tabanque, Revista Pedagógica, № 17. Pp. 87-98. Universidad de Valladolid, Valladolid.

Broccoli, A. (1977). “Antonio Gramsci y la educación como hegemonía”. Editorial Nueva Imagen, México.

Dómenech, J. (2003). "Las redes de Centros Educativos y la Renovación Pedagógica. La experiencia de la Coordinación de Escuelas 3-12 en Catalunya". Tabanque, Revista Pedagógica, $\mathrm{N}^{\circ} 17$. Pp. 99-110. Universidad de Valladolid, Valladolid.

Foucault, M. (1993). "Microfísica del Poder". Ediciones La Piqueta, Madrid.

Foucault, M. (2007). "Nacimiento de la Biopolítica. Curso en el College de France (1978-1979)". Fondo de Cultura Económica. Buenos Aires.

Habermas, J. (1987). "Teoría de la Acción Comunicativa”. Tomo II. Editorial Taurus. España.

Ibáñez, J. (2003). "Movimientos y Redes para una Cultura Transformadora". Tabanque, Revista Pedagógica, $\mathrm{N}^{\circ}$ 17. Pp. 11-32. Universidad de Valladolid, Valladolid.

Laraña, E., Gusfield, J. (1994). "Los Nuevos Movimientos Sociales: De la Ideología a la Identidad". Centro de Investigaciones Sociológicas (CIS). España.

Laraña, E. (1993). "Modelos de interpretación y cuestiones de método en el análisis de las migraciones españolas". En Laraña, E. y Gusfield, J. (eds.). Los Nuevos Movimientos Sociales: De la Ideología a la Identidad. Centro de Investigaciones Sociológicas (CIS). España.

Laraña, E. (1999). "La construcción de los Movimientos Sociales". Alianza Editorial. Madrid. España.

Llorente, M.A. (2003). "Los movimientos de Renovación Pedagógica y la lucha contra la mundialización neoliberal”. Tabanque, Revista Pedagógica, No 17. Pp. 71-86. Universidad de Valladolid, Valladolid.

Llorente, M.A. (2005). "El Futuro de la Renovación Pedagógica en la Escuela y de los MRPS desde la perspectiva de una Educación Comprometida". C.P. Cervantes. Federació de MPRs del País Valencia. Madrid, mayo 2005.

Mardones, J.M. (1996). "10 palabras clave sobre Movimientos Sociales”. Editorial Verbo Divino. España.

Martínez, J. (1990). "Renovación Pedagógica y Emancipación Profesional”. Editorial Universidad de Valencia Servicio de Publicaciones. España.

Melucci, A. (1994). “¿Qué hay de nuevo en los nuevos movimientos sociales?”. En Laraña, E. y Gusfield, J. (eds.). Los Nuevos Movimientos Sociales: De la Ideología a la Identidad. Centro de Investigaciones Sociológicas (CIS). España.

Retamozo, M. (2006). "Esbozos para una epistemología de los sujetos y los movimientos sociales". Cinta de Moebio: Revista Electrónica de Epistemología de Ciencias Sociales, ISSN 0717-554X, $N^{\circ}$ 26. Pp. 1-16. Santiago, Chile. 
Tomasi de Lampedusa, G. (2003). El Gatopardo. Cátedra, Letras Universales. España.

Touraine, A. (1987). El regreso del Actor (Le Retour de L'acteur), Editorial Universitaria de Buenos Aires, Argentina.

Zemelman, H. (1992). "Educación como construcción de sujetos sociales". La Piragua, Revista Latinoamericana de Educación y Política, No 5, CEAAL. Pp. 12-18. Santiago de Chile.

Zubero, I. (1996). "Movimientos Sociales y Alternativas de Sociedad". Ediciones Hoac. España. 
